Research Paper

\title{
Utility of Kynurenic Acid for Non-Invasive Detection of Metastatic Spread to Lymph Nodes in Non-Small Cell Lung Cancer
}

\author{
Dariusz Sagan ${ }^{1}$, Tomasz Kocki², Samir Patel ${ }^{3}$, Janusz Kocki ${ }^{4}$ \\ 1. Department of Thoracic Surgery, Medical University of Lublin, Jaczewskiego 8, 20-090 Lublin, Poland \\ 2. Department of Experimental and Clinical Pharmacology, Medical University of Lublin, Jaczewskiego 8, 20-090 Lublin, Poland \\ 3. English Division, IInd Medical Faculty, Medical University of Lublin, Poland \\ 4. Department of Clinical Genetics, Medical University of Lublin, Radziwiłłowska 11, 20-080 Lublin, Poland
}

$\triangle$ Corresponding author: Dariusz Sagan MD, PhD, FETCS, Department of Thoracic Surgery, Medical University of Lublin, Jaczewskiego 8, 20-090 Lublin, Poland. Phone: +48 50681320; e-mail: dariusz.sagan@umlub.pl

() Ivyspring International Publisher. This is an open-access article distributed under the terms of the Creative Commons License (http://creativecommons.org/ licenses/by-nc-nd/3.0/). Reproduction is permitted for personal, noncommercial use, provided that the article is in whole, unmodified, and properly cited.

Received: 2013.08.31; Accepted: 2014.01.20; Published: 2015.01.07

\begin{abstract}
Background: Kynurenic acid (KYNA) is a side-stream product of the kynurenine metabolic pathway that plays a controversial role in malignancies either enabling escape of malignant cells from immune surveillance or exerting antiproliferative effect on cancer cells, and is associated with differences in invasiveness related to metastatic spread to lymph nodes in lung cancer. Nodal involvement is a significant negative prognostic factor usually considered a contraindication for primary surgical resection.

Objective: To assess potential value of circulating KYNA for non-invasive identification of patients with metastatic lymph nodes $(\mathrm{N}+)$ in non-small cell lung cancer (NSCLC).

Methods: KYNA level in venous blood serum was determined with use of high performance liquid chromatography (HPLC) in 312 subjects including 230 patients with NSCLC and 32 healthy controls.

Results: Circulating KYNA level in NSCLC patients was higher than in controls $\left(93.6 \pm 61.9 \mathrm{pmol} / \mathrm{ml}\right.$ vs. $\left.31.4 \pm 16.6 \mathrm{pmol} / \mathrm{ml} ; \mathrm{p}=2.2 \cdot 10^{-15}\right)$ and positively correlated with $\mathrm{N}(\mathrm{R}=0.326$; $p=2 \cdot 10-6)$ but not with $T$ or $M$ stage $(p>0.05)$. In $N+$ patients it was higher than in N0 patients $\left(137.7 \pm 51.8 \mathrm{pmol} / \mathrm{ml}\right.$ vs. $\left.71.9 \pm 41.7 \mathrm{pmol} / \mathrm{ml} ; \mathrm{p}=4.8 \cdot 10^{-16}\right)$. KYNA effectively discriminated $\mathrm{N}+$ from N0 patients at a cut-off value $82.3 \mathrm{pmol} / \mathrm{ml}$ with sensitivity $94.7 \%(95 \% \mathrm{Cl} 87.1-98.5 \%)$, specificity $80.5 \%(95 \% \mathrm{Cl} 73.4-86.5 \%)$, negative predictive value $\mathrm{NPV}=96.8 \%, \mathrm{PPV}=70.5 \%$ and area under the ROC curve AUC=0.900 (95\% Cl 0.854-0.935; $p=0.0001)$.

Discussion and Conclusion: Circulating KYNA level measurement offers reliable non-invasive discrimination between $\mathrm{NO}$ and $\mathrm{N}+$ patients in NSCLC. Robust discriminatory characteristics of KYNA assay predestines it for clinical use as an adjunct facilitating selection of candidates for primary surgical resection.
\end{abstract}

Key words: diagnosis, immunology, marker, kynurenine

\section{Introduction}

Kynurenic acid (KYNA) is the end-stage product of the transamination side branch in the kynurenine pathway, which is the main pathway for tryptophan degradation in humans. This metabolic route consti- tutes the sole source of substrate for nicotinamide adenine dinucleotide (NAD+), participating in cellular energy supply via acetyl-CoA. Increased activation of tryptophan catabolism along this pathway has been 
identified as one of the factors contributing to suppression of specific anti-tumor immune response and to an escape of malignant cells from immune surveillance $[1,2,3]$. Kynurenine pathway metabolites induce numerous mechanisms used by malignant tumors to inhibit immune responses, including secretion of immunosuppresive cytokines, like IL-10 or TGF-beta, as well as stimulation of host cells to release immune inhibitors [4, 5]. Furthermore, kynurenines induce regulatory $\mathrm{T}$ cells (Treg) and impair dendritic cells (DCs) function contributing to immunosuppressive microenvironment that protects the tumor from host immunity [6].

Alterations in activity of kynurenine metabolic pathway have been detected in systemic malignancies and solid tumors [7, 8]. So far, in lung cancer, indoleamine 2,3-dioxygenase mRNA expression and serum tryptophan to kynurenine ratio have been investigated in small groups of patients $[9,10]$. Our recent results showed that KYNA may be associated with differences in invasiveness and biological behavior between adenocarcinoma and squamous cell lung cancer [11]. Results of experimental molecular studies identified KYNA as a ligand for G protein-coupled receptor 35 (GPR35) and for aryl hydrocarbon receptor (AHR), and revealed prominent expression of GPR35 and AHR both in immune tissues and in malignant cells $[12,13]$. The latter is especially pertinent in lung cancer, because it has been shown that AHR plays an important role in toxic response to cigarette smoke. Furthermore, GPR35 stimulated by KYNA has been suggested as a potential oncogene in gastric cancer [14]. Based on these data, we hypothesized that activation of kynurenine metabolic pathway resulting in altered levels of KYNA may be involved in pathogenesis and progression of non-small cell lung cancer (NSCLC).

Lung cancer is currently the most prevalent malignancy, and the most common cause of cancer mortality with approximately $1.38 \mathrm{mln}$ deaths worldwide. Despite systematic implementation of new diagnostic and therapeutic methods, the prognosis for this devastating disease is poor and further efforts to improve the outcomes of treatment are necessary [15, 16]. Among various therapeutic approaches, it is surgical resection that offers the best chance of complete cure in patients with NSCLC. However, metastatic involvement of mediastinal lymph nodes remarkably deteriorates prognosis in these patients and is widely considered a contraindication for surgical treatment. In such cases, combined regimens including chemotherapy are recommended as providing more beneficial results than primary surgical resection. Therefore, preoperative detection of metastatic lymph nodes is crucial for proper qualification of patients with
NSCLC for optimal treatment modality. Based on the above premises, we undertook efforts to identify kynurenine pathway metabolites that could be of potential usefulness as markers of lymph nodes involvement in NSCLC.

In the present study, we aimed to determine relationships between serum KYNA levels and TNM staging of NSCLC. Furthermore, we evaluated potential value of circulating KYNA to predict lymph nodes involvement in patients with NSCLC.

\section{Patients and methods}

A total of 312 subjects including 280 patients with radiologically detected pulmonary lesions suspected of lung cancer and referred to Thoracic Surgery Department for diagnosis or surgical treatment and 32 healthy volunteers were enrolled in the study between January 2008 and December 2010. In all patients venous blood samples were collected prior to any invasive procedures. Of these, 230 patients who subsequently were qualified for surgical procedures and in whom NSCLC was diagnosed constituted the study group, whereas 17 patients with small cell lung cancer and 33 patients with non-malignant tumors were excluded from the study. The patients in the study group were 154 men (67\%) and 76 women (33\%) at mean age $61.64 \pm 8.1$ ranging from 42 to 80 years. Detailed demographic and clinical characteristics of the study group are presented in table 1. Control group consisted of 32 healthy volunteers.

Table 1. Demographic and clinical characteristics of the study group

\begin{tabular}{lll}
\hline & Number of patients & $\begin{array}{l}\text { \% of the study } \\
\text { group }\end{array}$ \\
\hline Sex & \\
$\begin{array}{l}\text { Male } \\
\text { Female }\end{array}$ & 67 \\
Histology & 154 & 33 \\
Adenocarcinoma & 76 & \\
Squamous cell & 68 & 29.6 \\
Large cell & 95 & 41.3 \\
Mixed + undifferentiated & 36 & 15.7 \\
Staging & 31 & 13.4 \\
Ia & & \\
Ib & 62 & 27.0 \\
IIa & 69 & 29.9 \\
IIb & 39 & 17.0 \\
IIIa & 34 & 14.8 \\
IIIb & 19 & 8.3 \\
IV & 3 & 1.3 \\
Performance status & 4 & 1.7 \\
Karnofsky score & & \\
I00\% & & \\
$90 \%$ & 34 & 23.5 \\
$80 \%$ & 96 & 41.7 \\
$70 \%$ & 62 & 27.0 \\
Smoking history + & 16 & 7.8 \\
\hline & 174 & 75.6 \\
\hline
\end{tabular}


Venous blood samples for measurements were collected from peripheral vein in aseptic conditions, after at least 12 hours of fasting. Blood samples were centrifuged, and the separated serum samples were immediately deep frozen and stored at $-80^{\circ} \mathrm{C}$ until further analyses. After thawing, samples were acidified with trichloroacetic acid, and precipitated proteins were removed by centrifugation. The supernatants were analyzed for KYNA content by application to cation exchange Dowex 50W columns. Eluted KYNA was subjected to high performance liquid chromatography (HPLC) using Hewlett Packard 1050 HPLC system with C18 reverse phase column, and quantified fluorometrically (Hewlett Packard 1046A fluorescence detector).

Clinical and laboratory data were prospectively collected in a computer database. Staging was based on the pathologic assessment of resected specimens. The seventh edition of the lung cancer stage classification system was used for determination of pathologic staging in all patients in the study group [17]. Statistical analysis was performed using computer software Statistica 6.0 (StatSoft Polska Sp. z o.o., Krakow, Poland) and Medcalc 11 (MedCalc Software bvba, Mariakerke, Belgium). Results are presented as mean values \pm standard deviation (SD), median, minimum and maximum values, unless stated otherwise. Wilk-Shapiro test was used to assess normal distribution of values. U Mann-Whitney test was used for comparisons between two groups. Kruskall-Wallis ANOVA rank test with Dunn's post hoc test were used for comparisons between multiple groups. Probability $\mathrm{p}$ value less than 0.05 was considered statistically significant.

Diagnostic predictive performance was calculated using Receiver Operating Characteristics (ROC). Sample-size determinations were performed with an assumption of $a=5 \%$ and power $=80 \%$. The diagnostic performance of a new test was estimated as useful if an AUC of 0.75 could be obtained. A necessary sample size of 57 was calculated under these conditions with type I error 0.05 and type II error 0.2 .

The study has been approved by the Ethics Committee at our institution, and informed consent has been obtained from all participants prior to the enrollment in the study.

\section{Results}

\section{Serum KYNA levels in patients vs. healthy controls}

Serum KYNA level in the total NSCLC group was significantly higher than in healthy volunteers as controls $(93.6 \pm 61.9 \mathrm{pmol} / \mathrm{ml}$ vs. $31.4 \pm 16.6$, respectively; $\left.\mathrm{p}=2.2 \cdot 10^{-15}\right)$.

\section{Serum KYNA levels and TNM staging}

Serum KYNA level in patients with metastatic lymph nodes $\mathrm{N}+$ (including stages N1, N2, N3) was significantly higher than in patients with stage N0 $(137.7 \pm 51.8 \mathrm{pmol} / \mathrm{ml}$ vs. $71.9 \pm 41.7 \mathrm{pmol} / \mathrm{ml}$, respectively; $p=0.0001$ ) (Table 2, Figure 1 ). Post hoc test showed significant differences between groups N0 and N1, N0 and N2, and N0 and N3 ( $p=0.004 ; p=$ 0.00006 and $p=0.0058$, respectively). Differences between N1 and N2, N1 and N3, N2 and N3 were insignificant $(\mathrm{p}=0.4 ; \mathrm{p}=0.22$ and $\mathrm{p}=0.73$, respectively). Moreover, serum KYNA level showed positive correlation with $\mathrm{N}$ stage (Spearman rank correlation test, $\mathrm{R}=0.326 ; \mathrm{p}=2 \cdot 10^{-6}$ ) (Figure 2). KYNA level was not significantly correlated with the lymph nodes size ( $\mathrm{p}$ $=0.52$ ) or single $/$ multiple level N2 station lymph nodes involvement $(\mathrm{p}=0.38)$.

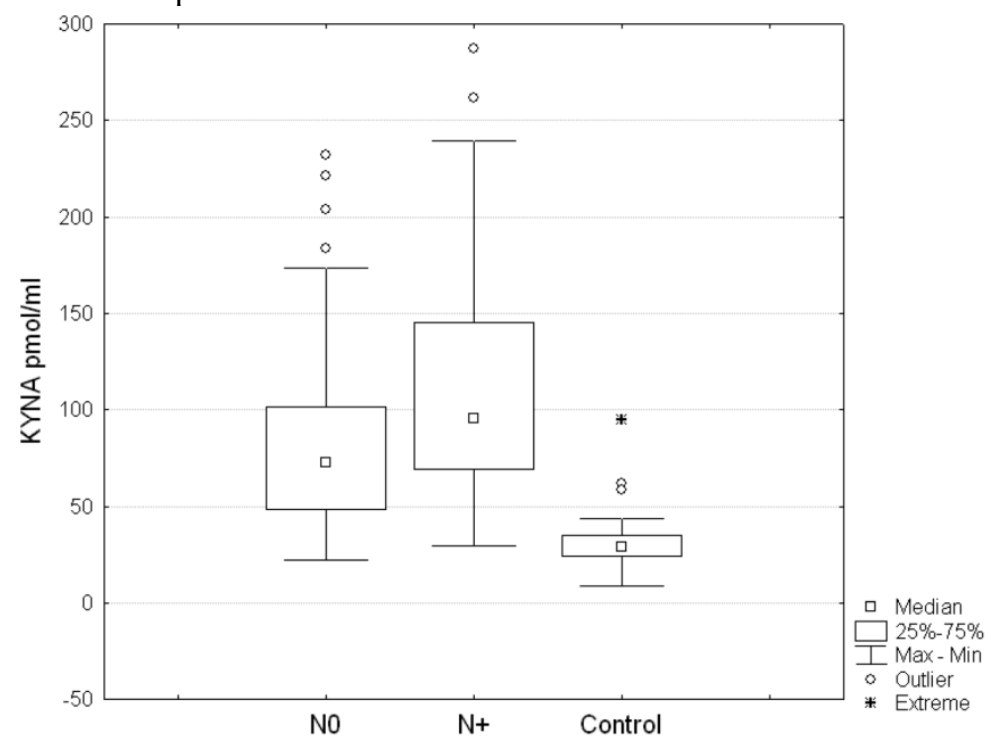

Fig 1. Serum KYNA level in patients with metastatic lymph nodes N+ (including stages N1, N2, N3) versus stage N0 ( $p=0.0001)$ and healthy controls. 
Table 2. Serum level of KYNA and metastatic lymph nodes involvement ( $N$ stage) in patients with NSCLC ( $p=0.0001$; post hoc tests between groups N0 and N1, N0 and N2, and N0 and N3 $p=0.004 ; p=0.00006$ and $p=0.0058$, respectively)

\begin{tabular}{|c|c|c|c|c|c|c|}
\hline KYNA & No & $\begin{array}{l}\mathrm{N}+ \\
\text { (incl. N1, N2, N3) }\end{array}$ & N1 & N2 & N3 & Controls \\
\hline Mean (pmol/ml) & 71.9 & 137.7 & 116.6 & 155.8 & 150.6 & 31.4 \\
\hline Standard deviation (pmol/ml) & 41.7 & 51.8 & 47.9 & 74.3 & 70.6 & 16.6 \\
\hline $95 \% \mathrm{CI}(\mathrm{pmol} / \mathrm{ml})$ & $71.0-85.2$ & $99.6-142.0$ & $92.0-131.2$ & $94.2-181.5$ & $62,9-238,3$ & $25.4-37.4$ \\
\hline $\mathbf{n}$ & 153 & 77 & 58 & 17 & 2 & 32 \\
\hline
\end{tabular}

Table 3. Serum level of KYNA in relation to stage groups of patients with NSCLC (ANOVA rank Kruskall - Wallis test: $H=14.99 ; p=$ $0.0203)$

\begin{tabular}{|c|c|c|c|c|c|c|c|}
\hline & IA & IB & IIA & IIB & IIIA & IIIB & IV \\
\hline Mean (pmol/ml) & 86.08 & 78.72 & 87.21 & 77.96 & 107.14 & 180.09 & 106.88 \\
\hline Standard deviation (pmol/ml) & 49.89 & 33.71 & 43.85 & 39.48 & 66.87 & 67.81 & 65.25 \\
\hline $95 \% \mathrm{CI}(\mathrm{pmol} / \mathrm{ml})$ & $68.39-103.77$ & $69.43-88.01$ & $74.48-99.95$ & $61.29-94.63$ & $81.70-132.57$ & $123.41-236.78$ & $67.45-146.31$ \\
\hline Number of patients & 62 & 69 & 39 & 34 & 19 & 3 & 4 \\
\hline
\end{tabular}

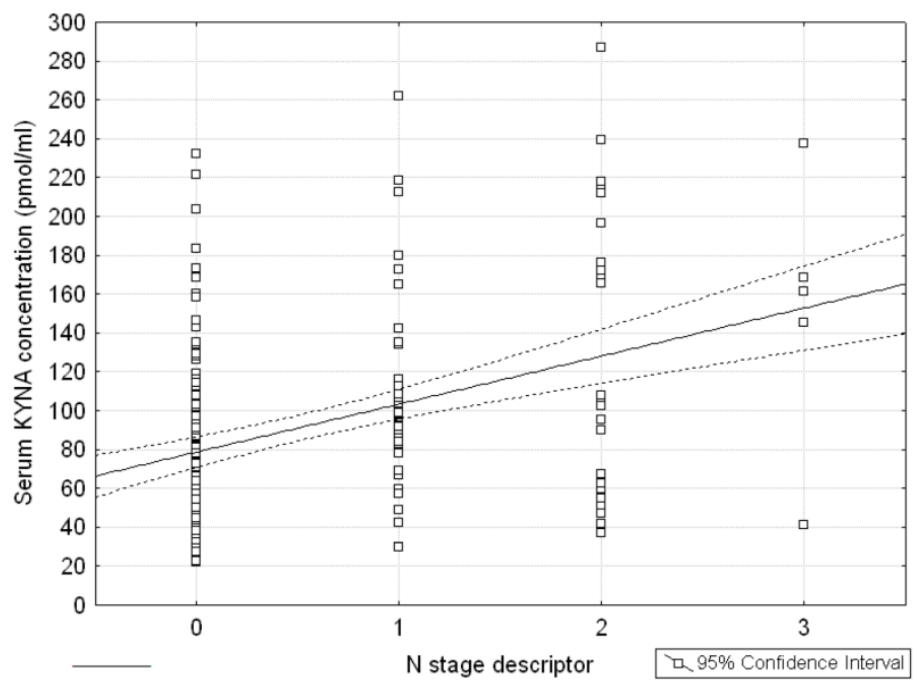

Fig. 2. Correlation between $N$ stage descriptor and serum KYNA level in patients with NSCLC; Spearman rank correlation test: $R=0.326 ; p=2 \cdot 10^{-6}$

No statistically significant differences were disclosed in relation to either $\mathrm{T}$ or $\mathrm{M}$ descriptor (ANOVA rank Kruskall - Wallis test $\mathrm{H}=7.5 ; \mathrm{p}=0.18$, and $\mathrm{H}=$ 1.82; $\mathrm{p}=0.4$, respectively) (Figures 3 and 4). Serum concentration of KYNA showed no significant correlation with the largest dimension of the tumor as a continuous variable either (Spearman correlation test $\mathrm{R}=0.069 ; \mathrm{p}=0.326$ ).

Serum KYNA level showed significant differences between patients at various stages of the disease according to stage groupings (ANOVA rank Kruskall - Wallis test: $\mathrm{H}=14.99 ; \mathrm{p}=0.0203$ ). Post hoc test showed that serum KYNA concentration in patients with stage IIIB was significantly higher compared to patients with stage IA, IB, IIA, IIB, IIIA or IV (180.09 \pm $67.81 \mathrm{pmol} / \mathrm{ml}$ vs. $86.08 \pm 49.89 \mathrm{pmol} / \mathrm{ml} \mathrm{p}=0.0021$, $78.72 \pm 33.71 \mathrm{pmol} / \mathrm{ml} \mathrm{p}=0.0006 ; 87.21 \pm 43.85$ $\mathrm{pmol} / \mathrm{ml} \mathrm{p}=0.0025 ; 77.96 \pm 39.48 \mathrm{pmol} / \mathrm{ml} \mathrm{p}=$ $0.0005 ; 107.14 \pm 66.87 \mathrm{pmol} / \mathrm{ml} \mathrm{p}=0.0422$, and 106.88 $\pm 65.25 \mathrm{pmol} / \mathrm{ml} \mathrm{p}=0.0409$, respectively) (Table 3,
Figure 5). The remaining differences were insignificant. Furthermore, serum KYNA level positively correlated with the stage of the disease (Spearman correlation test $R=0.153 ; p=0.027$, Figure 6).

\section{Receiver Operating Characteristic (ROC) analysis of N0 vs. $\mathbf{N}+$ patients}

ROC analysis revealed that optimal diagnostic accuracy of serum KYNA assay for discrimination between $\mathrm{N} 0$ and $\mathrm{N}+$ patients was achieved at a cut-off value $82.3 \mathrm{pmol} / \mathrm{ml}$ (Fig 7). At this criterion value the test had sensitivity $94.7 \%$ (95\% CI 87.1 to $98.5 \%)$, specificity $80.5 \%$ (95\% CI 73.4 to $86.5 \%$ ), negative predictive value (NPV) $96.8 \%$ (95\% CI 92.2 to $99.1 \%$ ), positive predictive value (PPV) 70.5\% (95\% CI 60.6 to 79.2\%), positive likelihood ratio (PLR) 4.86 (95\% CI 3.5 to 6.7), negative likelihood ratio (NLR) 0.07 (95\% CI 0.03 to 0.2), and area under the ROC curve (AUC) 0.900 (95\% CI 0.854 to $0.935 ; \mathrm{P}=0.0001$ ). 


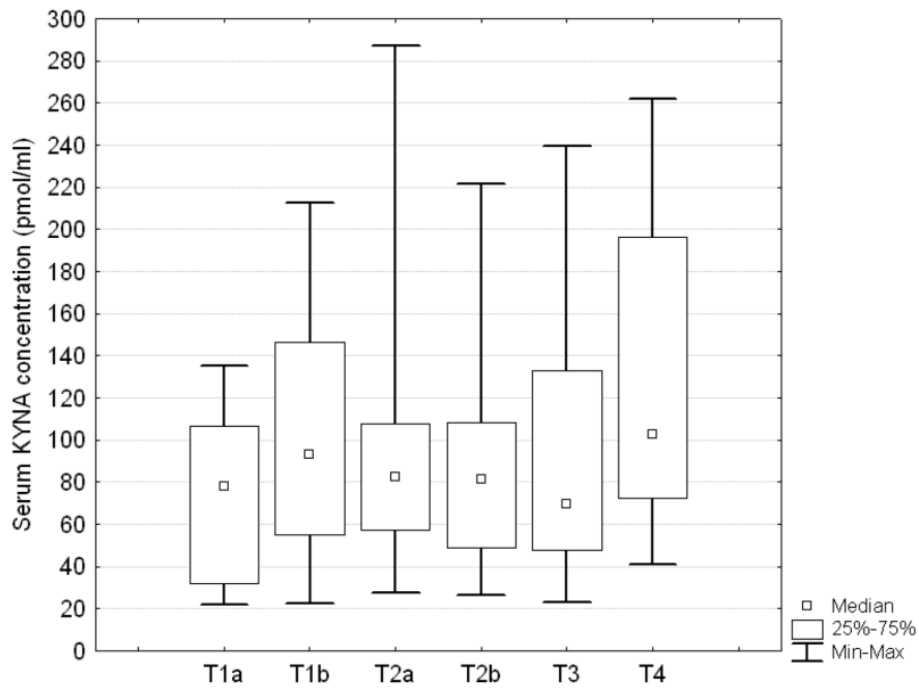

Fig. 3. Serum KYNA level in relation to T stage descriptor in patients with NSCLC; Kruskall - Wallis test: $H=7.5 ; p=0.18$

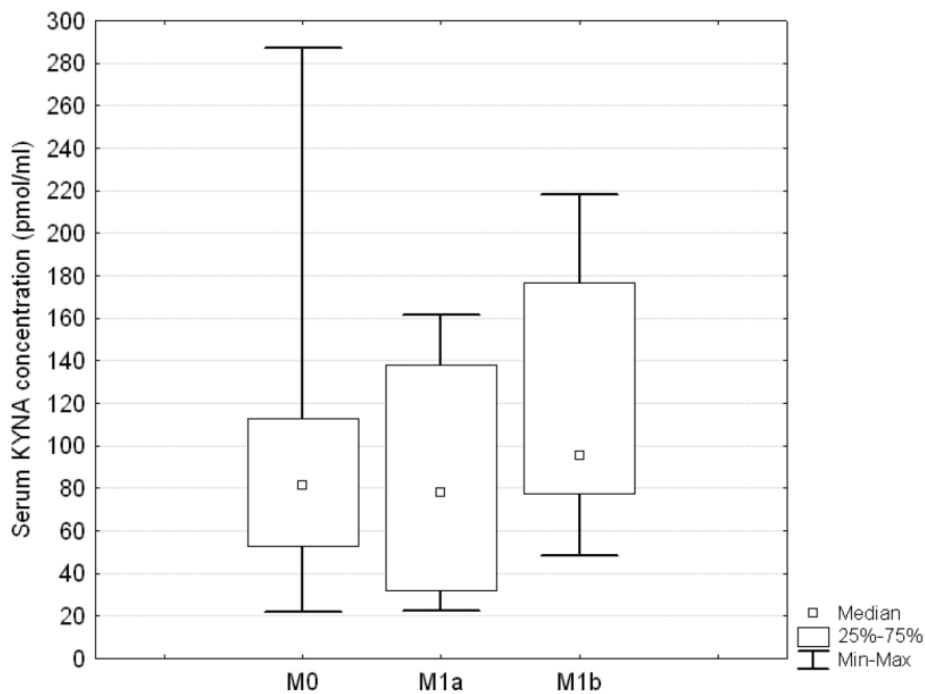

Fig. 4. Serum KYNA level in relation to M stage descriptor in patients with NSCLC; no statistically significant differences between the groups; Kruskall Wallis test: $\mathrm{H}=1.82 ; \mathrm{p}=0.4$

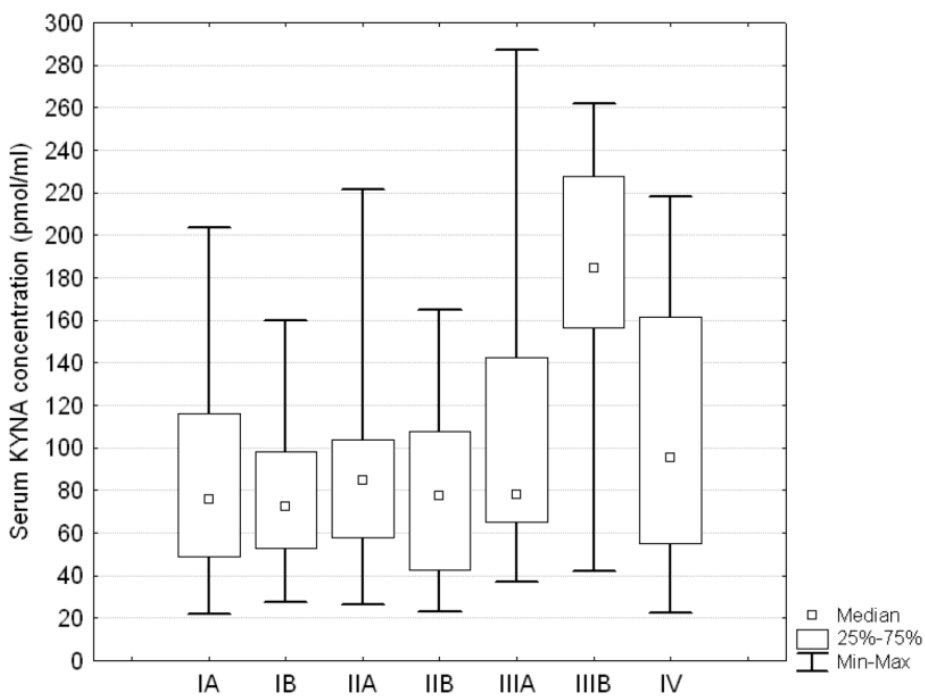

Fig. 5. Serum KYNA level in relation to stage groupings in patients with NSCLC; Kruskall - Wallis test $H=14.99 ; p=0.0203$; Post hoc test showed significant differences between IIIB and IA, IB, IIA, IIB, IIIA or IV $(p=0.0021, p=0.0006 ; p=0.0025 ; p=0.0005 ; p=0.0422$, and $p=0.0409$, respectively) 


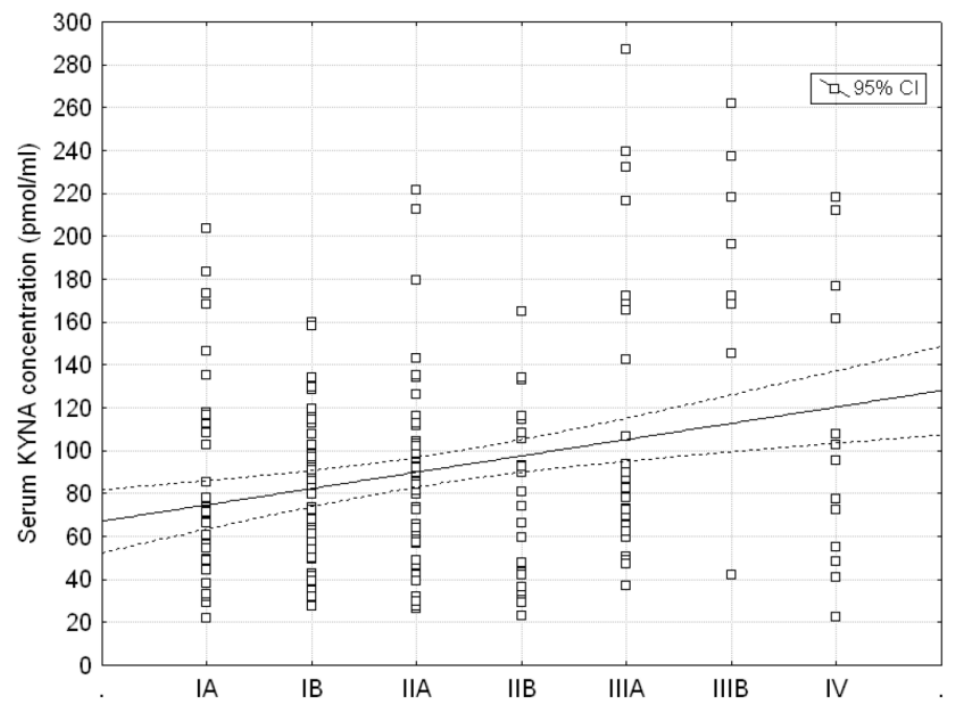

Fig. 6. Correlation between stage groupings and serum KYNA level in patients with NSCLC; Spearman rank correlation test: $R=0.153 ; p=0.027$

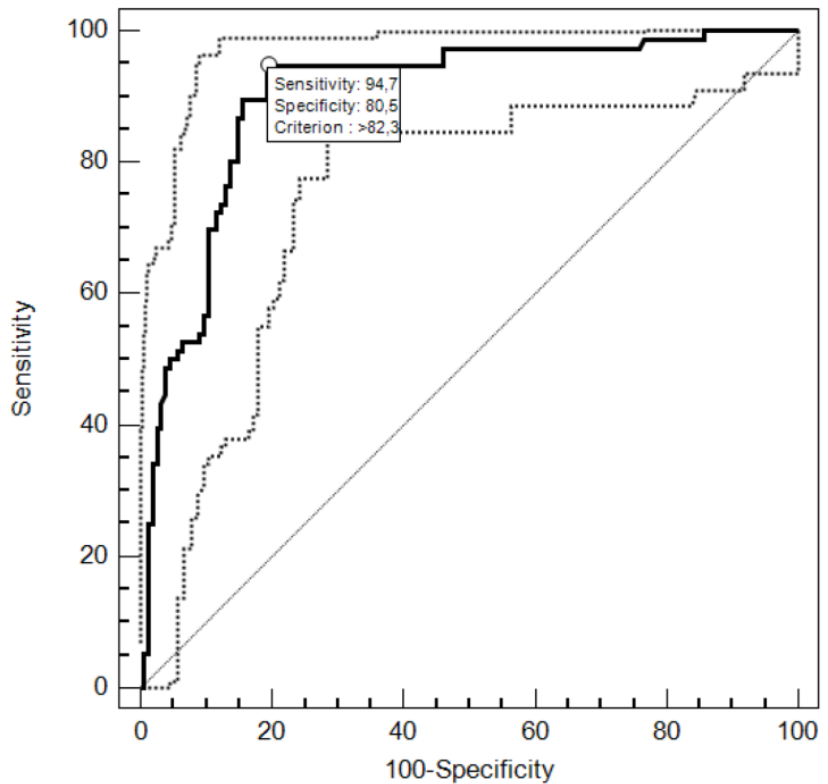

Fig. 7. Receiver Operating Characteristic (ROC) analysis of serum KYNA test performance for preoperative discrimination between N0 vs. N+ patients

\section{Comments}

In the current study we have demonstrated that serum KYNA level measurement reliably discriminated metastatic involvement of lymph nodes in patients with NSCLC. The serum KYNA level in N+ patients was significantly higher than in N0 patients. ROC analysis indicated a cut-off value of 82.3 $\mathrm{pmol} / \mathrm{ml} \mathrm{KYNA}$ as an optimal criterion discriminating between $\mathrm{N} 0$ and $\mathrm{N}+$ disease with sensitivity $94.7 \%$. Furthermore, our findings revealed that serum KYNA level in patients NSCLC was significantly higher than in healthy controls and positively correlated with $\mathrm{N}$ status and with stage grouping.

Very few studies have been published so far on the role of kynurenine metabolic pathway in lung cancer. Our recent results showed that KYNA may be associated with differences in invasiveness and in biological behavior between adenocarcinoma and squamous cell lung cancer [11]. Single reports have been published on KYNA in other malignancies. Increased plasma and bone marrow KYNA concentrations have been detected in monoclonal gammopathy of undetermined significance and multiple myeloma patients [7]. Some authors reported overall activation of kynurenine metabolic route in malignant diseases. Increased tryptophan catabolism was detected in adult T-cell leukemia, gynecological tumors and colorectal cancers $[8,18,19]$. Tryptophan degradation via the kynurenine metabolic pathway has been identified as a remarkable factor contributing to an escape of tumor cells from immune surveillance [20]. Prod- 
ucts of this metabolic route suppress antitumor responses and induce an immunoregulatory or an anergic $\mathrm{T}$ cell phenotype at a systemic level $[3,8]$. Furthermore, Wang and colleagues demonstrated that KYNA inhibits lipopolisaccharide-induced tumor necrosis factor-a secretion in peripheral blood mononuclear cells [12].

Association between involvement of lymph nodes and elevated KYNA levels, demonstrated in our study, is a remarkable finding, as lymph nodes constitute an important part of the human immune system. It further supports a hypothesis that increased activity of kynurenine pathway is involved in the progression of the malignant disease, possibly through immunosuppressive effect. However, an increase in endogenous production of interferon- $\gamma$ $($ IFN- $\gamma)$, which is the most potent inducer of IDO, may be another explanation of this phenomenon. Enhanced tryptophan metabolism by interferon-induced pulmonary IDO has been demonstrated in human lungs bearing cancer, and suggested as a unique host defense mechanism [20]. This may be considered a part of a wider systemic mechanism of inflammatory reaction. In several cancers, elevated kynurenine metabolic route activity has been attributed to locally increased levels of IFN- $\gamma$, in particular in macrophages and dendritic cells [8, 19, 21, 22]. In contrast, neither elevation of serum IFN- $\gamma$ level nor correlation between kynurenine/tryptophan ratio and IFN- $\gamma$ level have been detected in patients with lung cancer [9].

Experimental studies revealed antiproliferative effect of KYNA at micro- and milimolar concentrations against colon cancer cells [23]. This finding suggests that KYNA may have a potential anti-tumor activity and be utilized by immune system as an anti-cancer agent. It might explain elevated serum levels of KYNA in advanced stages of cancer. Similarly, it was suggested that IFN-mediated induction of IDO takes place in human lung parenchyma as a response to cancer, leading to metabolic consequences such as depletion of tryptophan and accumulation of kynurenine, which may provide a unique host defense mechanism [24]. A hypothesis of a feedback control loop may be another speculation explaining the rise in KYNA levels in advanced stages of malignant diseases. According to this theory, overstimulation of the immune system results in increased KYNA production, which, in turn, downregulates the immune system in a mechanism similar to a feedback loop providing control over the entire system [7]. Further research will be required to determine specific mechanisms of kynurenine route activation in cancer patients.

To date, few studies dealt with tryptophan deg- radation along kynurenine pathway in patients with NSCLC. Suzuki and colleagues reported increased enzymatic activity of kynurenine metabolic pathway in advanced stages of lung cancer [9], which is consistent with our findings. The authors measured serum kynurenine and tryptophan concentrations and estimated indoleamine 2,3-dioxygenase (IDO) activity by calculating the kynurenine to tryptophan ratio. They found that patients with advanced lung cancer had significantly higher IDO activity than those at early stages, indicating a correlation with progression of the disease. Kynurenine/tryptophan ratio was significantly higher in N3 than N0 or N2, suggesting that higher IDO activity is associated with the extent of lymph node metastasis. Neither $\mathrm{T}$ nor $\mathrm{M}$ descriptors were related to IDO activity.

In contrast, Karanikas and colleagues found no significant correlation between disease staging and IDO gene expression in tumor tissues using quantitative real-time polymerase chain reaction in 28 patients with NSCLC [10]. However, increased level of IDO mRNA expression in tumor tissue, compared to normal lung tissue was disclosed. Besides, they demonstrated IDO mRNA constitutively expressed by lung cancer cells, but attributed the production of the enzyme also to other cells recruited in the tumor micro-environment and the peri-tumoral lung area.

Currently, in patients with NSCLC, N2 involvement is considered an indication for neoadjuvant chemotherapy, and there is a tendency among oncologists to extend this regimen onto N1 cases. This is one of the reasons why preoperative detection of metastatic lymph nodes in NSCLC patients is crucial for adequate selection of candidates for resection or other treatment modalities. However, despite clinical, bronchoscopic and imaging examinations, it remains a difficult task $[25,26]$. Our findings indicate that elevated serum KYNA level may be considered a biomarker of metastatic lymph nodes involvement. In conjunction with clinical assessment, computed tomography (CT) and positron emission tomography (PET) it may facilitate preoperative determination of $\mathrm{N}+$ stage in NSCLC allowing for more precise matching of patients for optimal treatment modalities.

Our study has some limitations. KYNA measurement requires application of advanced laboratory HPLC techniques as there are no clinically available instant tests for this metabolite yet. Relatively low number of patients in some subgroups resulted in a considerable variance in KYNA levels within the stratified groups of patients. Further research on the relevance of the kynurenine pathway activity in lung cancer is warranted and it will be facilitated by the advent of easy-to-use clinical tests for KYNA.

Concluding, our study demonstrates that circu- 
lating KYNA level measurement offers reliable preoperative non-invasive discrimination between N0 and $\mathrm{N}+$ patients in NSCLC. Robust discriminatory characteristics of KYNA assay predestines this test for clinical use as an adjunct facilitating selection of candidates for primary surgical resection or for other treatment modalities.

\section{Competing Interests}

The authors have declared that no competing interest exists.

\section{References}

1. Munn DH, Mellor AL. Indoleamine 2,3-dioxygenase and tumor-induced tolerance. J Clin Invest. 2007, 117:1147-1154.

2. Munn DH, Sharma MD, Hou D, et al. Expression of indoleamine 2,3-dioxygenase by plasmacytoid dendritic cells in tumor-draining lymph nodes. J Clin Invest. 2004, 114:280-290.

3. Mellor AL, Keskin DB, Johnson $\mathrm{T}$, et al. Cells expressing indoleamine 2,3-dioxygenase inhibit T cell responses. J Immunol. 2002, 168:3771-3776.

4. Dubinett S, Sharma S. Towards effective immunotherapy for lung cancer: simultaneous targeting of tumor-initiating cells and immune pathways in the tumor microenvironment. Immunotherapy. 2009;1:721-725.

5. Halak BK, Maguire HC, Jr., Lattime EC. Tumor-induced interleukin-10 inhibits type 1 immune responses directed at a tumor antigen as well as a non-tumor antigen present at the tumor site. Cancer Res. 1999, 59:911-917.

6. Zou W. Immunosuppressive networks in the tumour environment and their therapeutic relevance. Nat Rev Cancer. 2005; 5:263-274.

7. Zdzisinska B, Wejksza K, Walter-Croneck A, et al. Kynurenic acid in blood and bone marrow plasma of monoclonal gammopathy of undetermined significance (MGUS) and multiple myeloma (MM) patients. Leuk Res. 2010; 34:38-45.

8. Schroecksnadel K, Winkler C, Fuith LC, Fuchs D. Tryptophan degradation in patients with gynecological cancer correlates with immune activation. Cancer Lett. 2005; 223:323-329.

9. Suzuki Y, Suda T, Furuhashi K, et al. Increased serum kynurenine/tryptophan ratio correlates with disease progression in lung cancer. Lung Cancer. 2012; 67:361-365.

10. Karanikas V, Zamanakou M, Kerenidi T, et al. Indoleamine 2,3-dioxygenase (IDO) expression in lung cancer. Cancer Biol Ther. 2007; 6:1258-1262.

11. Sagan D, Kocki T, Kocki J, Szumilo J. Serum Kynurenic Acid: Possible Association with Invasiveness of Non-small Cell Lung Cancer. Asian Pac J Cancer Prev. 2012; 13:4241-4.

12. Wang J, Simonavicius $\mathrm{N}, \mathrm{Wu} \mathrm{X}$, et al. Kynurenic acid as a ligand for orphan $\mathrm{G}$ protein-coupled receptor GPR35. J Biol Chem. 2006; 281:22021-22028.

13. DiNatale BC, Murray IA, Schroeder JC, et al. Kynurenic acid is a potent endogenous aryl hydrocarbon receptor ligand that synergistically induces interleukin-6 in the presence of inflammatory signaling. Toxicol Sci. 2010; 115:89-97.

14. Okumura S, Baba H, Kumada T, et al. Cloning of a G-protein-coupled receptor that shows an activity to transform NIH3T3 cells and is expressed in gastric cancer cells. Cancer Sci. 2004; 95:131-135.

15. Sant M, Allemani C, Santaquilani M, et al. EUROCARE-4. Survival of cancer patients diagnosed in 1995-1999. Results and commentary. Eur J Cancer. 2009; 45:931-991.

16. Verdecchia A, Guzzinati S, Francisci S, et al. Survival trends in European cancer patients diagnosed from 1988 to 1999 . Eur J Cancer. 2009; 45:1042-1066.

17. Detterbeck FC, Boffa DJ, Tanoue LT. The new lung cancer staging system. Chest. 2009; 136:260-271.

18. Hoshi $\mathrm{M}$, Ito $\mathrm{H}$, Fujigaki $\mathrm{H}$, et al. Indoleamine 2,3-dioxygenase is highly expressed in human adult T-cell leukemia/lymphoma and chemotherapy changes tryptophan catabolism in serum and reduced activity. Leuk Res. 2009; 33:39-45.

19. Huang A, Fuchs D, Widner B, et al. Serum tryptophan decrease correlates with immune activation and impaired quality of life in colorectal cancer. Br J Cancer. 2002; 86:1691-1696.

20. Munn DH, Shafizadeh E, Attwood JT, et al. Inhibition of T cell proliferation by macrophage tryptophan catabolism. J Exp Med. 1999; 189:1363-1372.

21. Schroecksnadel K, Fiegl M, Prassl K, et al. Diminished quality of life in patients with cancer correlates with tryptophan degradation. J Cancer Res Clin Oncol. 2007; 133:477-485.

22. Weinlich G, Murr C, Richardsen L, et al. Decreased serum tryptophan concentration predicts poor prognosis in malignant melanoma patients. Dermatology. 2007; 214:8-14.

23. Walczak K, Dabrowski W, Langner E, et al. Kynurenic acid synthesis and kynurenine aminotransferases expression in colon derived normal and cancer cells. Scand J Gastroenterol. 2011; 46(7-8):903-12.
24. Yasui H, Takai K, Yoshida R, Hayaishi O. Interferon enhances tryptophan metabolism by inducing pulmonary indoleamine 2,3-dioxygenase: its possible occurrence in cancer patients. Proc Natl Acad Sci U S A. 1986; 83:6622-6626.

25. Gomez-Caro A, Garcia S, Reguart N, et al. Incidence of occult mediastinal node involvement in cNO non-small-cell lung cancer patients after negative uptake of positron emission tomography/computer tomography scan. Eur J Cardiothorac Surg. 2010; 37:1168-1174.

26. Passlick B, Izbicki JR, Kubuschok B, et al. Detection of disseminated lung cancer cells in lymph nodes: impact on staging and prognosis. Ann Thorac Surg. 1996; 61:177-182. 